hematopoietic elements and expression of cyclin $D_{1}$ were not significantly associated with progression to symptomatic disease.

The authors conclude that patients with smoldering multiple myeloma should be closely followed up, with laboratory tests every 4-6 months once stable asymptomatic disease has been confirmed.

Original article Kyle RA et al. (2007) Clinical course and prognosis of smoldering (asymptomatic) multiple myeloma. N Engl J Med 356: 2582-2590

\section{STAT3 polymorphism: a potential marker of response to IFN- $\alpha$ therapy in metastatic RCC}

Interferon (IFN)- $\alpha$ immunotherapy has been used in patients with metastatic renal cell carcinoma (MRCC), as conventional chemotherapy is ineffective in this setting. Rates of response to IFN- $\alpha$ immunotherapy are, however, low and there is a need for markers to predict which patients will benefit from this treatment. In order to identify such a marker, Ito and colleagues carried out a comprehensive association study in a population of Japanese patients who had been treated with IFN- $\alpha$ for MRCC.

The authors investigated single nucleotide polymorphisms (SNPs) in 33 candidate genes in 75 patients, 29 of whom responded to IFN- $\alpha$. Seventeen SNPs were found to be associated with IFN- $\alpha$ response $(P \leq 0.1)$. Stepwise logistic regression analysis identified 11 SNPs that predicted better response to IFN- $\alpha$. The strongest predictors were in the STAT3 gene. Extensive sequencing of STAT3 found rs4796793, located in the 5' flanking region, to be most strongly associated with response to IFN- $\alpha$. The rs4796793 SNP did not directly influence STAT3 expression, but did correlate with STAT3 mRNA level. The minor (C) allele was associated with lower STAT3 expression, and also tended to occur relatively more frequently in IFN- $\alpha$ responders. Further in vitro experiments revealed that suppression of STAT3 enhanced cellular sensitivity to IFN- $\alpha$ administration.

STAT3 polymorphism could be used as a marker of response to IFN- $\alpha$ immunotherapy in MRCC. Ito et al. are currently evaluating the utility of rs4796793 as a marker of IFN- $\alpha$ response in a prospective Japanese cohort.

Original article Ito N et al. (2007) STAT3 polymorphism predicts interferon-alfa response in patients with metastatic renal cell carcinoma. J Clin Oncol 19: 2785-2791

\section{Adjuvant radiation therapy can improve survival in gallbladder adenocarcinoma}

Gallbladder cancer is associated with poor outcomes, and the rates of disease recurrence after curative resection are high. To date, the optimum role of adjuvant radiation in this setting is unclear. A recent analysis of data from the US Surveillance, Epidemiological, and End Results (SEER) program has addressed this issue.

Mojica and co-workers identified 1,799 cases of locoregional gallbladder adenocarcinoma treated surgically during the period 1992-2002. The majority of cases were treated with simple cholecystectomy. Multivariate analysis revealed sex, age, tumor grade, tumor stage, and use of adjuvant radiation therapy to be independent predictors of overall survival. The role of adjuvant chemotherapy could not be assessed because the SEER database did not report the use of this modality.

In total, 369 (21\%) patients received adjuvant radiation therapy. Compared with the patients who did not receive adjuvant radiation therapy, those in the adjuvant-radiation group were younger and had higher disease stage at presentation. Recipients of adjuvant radiotherapy demonstrated a median overall survival of 14 months, compared with 8 months in those who did not receive any adjuvant radiotherapy $(P<0.0001)$. This survival advantage was also found in the subgroups of patients with regional disease (16 vs 5 months; including regional disease treated with extended cholecystectomy [13 vs 6 months]) and local invasion of the liver (14 vs 11 months). Adjuvant radiation was not, however, associated with improvements in overall survival in patients presenting with stage I disease limited to the gallbladder.

Original article Mojica P et al. (2007) Adjuvant radiation therapy is associated with improved survival for gallbladder carcinoma with regional metastatic disease. J Surg Onc 96: $8-13$ 